\title{
Effects of Aerobic Exercise Training on the Stiffness of Central and Peripheral Arteries in Middle-Aged Sedentary Men
}

\author{
Koichiro HAYASHI*, Jun SUGAWARA*, Hidehiko KOMINE*, Seiji MAEDA ${ }^{\dagger}$, and \\ Takashi YOKOI* \\ *Institute for Human Science and Biomedical Engineering, National Institute of Advanced Industrial Science and \\ Technology (AIST), and ${ }^{\dagger}$ Center for Tsukuba Advanced Research Alliance (TARA), University of Tsukuba, Tsu- \\ kuba, Ibaraki, 305-8566 Japan
}

\begin{abstract}
Aortic pulse wave velocity (PWV) significantly decreased after 16 weeks of moderate-intensity exercise training (walking/jogging) in 17 sedentary middle-aged men, whereas leg PWV did not. These results suggest that
\end{abstract}

in contrast with central arterial stiffness, peripheral arterial stiffness is difficult to change with aerobic exercise training. [The Japanese Journal of Physiology 55: 235-239, 2005]

Key words: arterial stiffness, pulse wave velocity, aerobic exercise training.

Central elastic arteries (e.g., the aorta and carotid artery) have high compliance and take the role of a buffering function damping down the fluctuation in arterial pressure [1]. The loss of this function is associated with future cardiovascular disease [2]. Peripheral arteries (e.g., brachial and femoral arteries) also act as a buffer of pulsatile blood flow at its input and efficiently absorb the energy during the systolic component of pulsatile blood flow, which reduces the energy loss by making the blood flow smoothly [1].

It has been reported that moderate-intensity aerobic exercise training can reduce central arterial stiffness in healthy sedentary people $[3,4]$, whereas the effects of the exercise training on peripheral arterial stiffness have never been conclusive. Tanaka et al. [4] indicated that arterial stiffness (via pulse-wave velocity [PWV]) of the upper and lower limbs in well-trained postmenopausal female runners was not different from that in sedentary counterparts. In contrast, Schmidt-Trucksuss et al. [5] indicated that femoral arterial lumen diameter and compliance (via an ultrasound imaging device and simultaneous applanation tonometry) in endurance-trained males were significantly higher than those in age-matched sedentary counterparts.
This discrepancy seems to be derived from differences in the evaluation of arterial stiffness (e.g., PWV or ultrasound imaging) and in the context of exercise training (e.g., intensity and/or duration).

Dinneno et al. [6] observed an expansion of the femoral arterial lumen diameter in previously sedentary middle-aged and elderly men after a 3-month aerobic exercise intervention (primarily walking). The expansion of arteries seems to relate to structural remodeling or reduced vascular smooth muscular tone and leads to decreasing peripheral arterial stiffness. Moreover, it has been suggested that acute moderateintensity cycling reduces central and peripheral arterial stiffness [7]. With this information as background, the aim of the present study was to test the hypothesis that in middle-aged men, moderate-intensity aerobic exercise training (walking/jogging) reduces not only central arterial stiffness, but also peripheral arterial stiffness.

\section{Methods}

Subjects. We studied 17 healthy and sedentary middle-aged men, aged 31-64 years (mean age: $50 \pm 3$ yr). All subjects were normotensive $(<140 / 90 \mathrm{mmHg})$,

Received on Jun 2, 2005; accepted on Oct 26, 2005; released online on Oct 27, 2005; DOI: 10.2170/jjphysiol.S2116

Correspondence should be addressed to: Koichiro Hayashi, Institute for Human Science and Biomedical Engineering, National Institute of Advanced Industrial Science and Technology (AIST), 1-1-1 Higashi, Tsukuba, Ibaraki, 305-8566 Japan. Tel: +81-298-61-6663, Fax: +81-298-61-6660, E-mail: k-hayashi@aist.go.jp 
nonobese (BMI $<30 \mathrm{~kg} / \mathrm{m}^{2}$ ), and nonsmokers. They were free of medication and of overt chronic heart and lung disease as assessed by medical history, and all gave their written informed consent to participate. All procedures were reviewed and approved by the Human Research Committee of the National Institute of Advanced Industrial Science and Technology (AIST).

Measurements. Training intervention for individuals was done from May to November. All measurements were performed in a quiet air-conditioned room $\left(24-25^{\circ} \mathrm{C}\right)$ at the same time of day in the morning before and after the exercise-training intervention. To avoid the effects of acute exercise, the subjects were directed to perform neither training nor other vigorous physical activity on the day before measurements. The subjects also abstained from caffeine and fasted for 12 $\mathrm{h}$ overnight before the measurements.

Brachial and ankle blood pressures and heart rate were measured after a supine resting of at least 15 min by a semiautomated device (form PWV/ABI, Colin MT, Komaki, Japan), as previously described [8]. For the measurement of PWV, pressure waveforms at carotid, femoral, and posterior-tibial arteries were simultaneously recorded. Carotid and femoral arterial pressure waveforms were measured by two multielement tonometry sensors manually attached to the left carotid and left femoral arteries. Posterior-tibial arterial pressure waveforms were recorded by a cuff connected to a pletysmographic sensor wrapped around the left ankle. The PWV was calculated from the distance between two arterial recording sites divided by the time delay between the proximal and distal "foot" waveforms, i.e., between the carotid and femoral arteries (aortic PWV) and between the femoral and posterior-tibial arteries (leg PWV).

The common carotid and femoral arterial lumen diameter and compliance, the inverse nature of arterial stiffness, were evaluated, as we previously described [9]. Briefly, the B-mode longitudinal ultrasound images of the right common carotid and the left common femoral arteries were recorded via an ultrasound device with a high-resolution $(10 \mathrm{MHz})$ linear transducer and analyzed with image-analysis software (SonoSite180PLUS; SonoSite Inc., Bothell, WA). The diameters were measured from the intima of the far wall to the media-adventitia of the near wall at 1 to $2 \mathrm{~cm}$ proximal to the bifurcation of the right common carotid artery and at 2 to $3 \mathrm{~cm}$ proximal to the bifurcation of the left common femoral artery. The carotid and femoral arterial compliance were calculated by use of the equations $\left[\left(D_{1}-D_{0}\right) / D_{0}\right] /\left[2\left(P_{1}\right.\right.$
$\left.\left.-P_{0}\right)\right] \times \pi \times\left(D_{0}\right)^{2}$, where $D_{1}$ and $D_{0}$ are the maximal and minimum diameters and $P_{1}$ and $P_{0}$ are the highest and lowest arterial pulse pressure.

The carotid arterial pressure was recorded using an applanation tonometry probe incorporating a highfidelity strain-gauge transducer (SPT-301, Millar Instruments, Houston, TX) on the left common carotid artery, as we previously described [9]. Because the baseline levels of carotid blood pressure are subject to hold-down forces, the pressure signal obtained by tonometry was calibrated by equilibrating the carotid mean arterial and diastolic blood pressures to the brachial mean and diastolic blood pressures measured by an oscilometric device. To calculate femoral artery compliance, we used the blood pressure values obtained at the posterior tibial artery.

To measure the maximal oxygen consumption $\left(V \mathrm{O}_{2 \max }\right)$, all subjects underwent an incremental cycle exercise test (after $2 \mathrm{~min}$ at $20 \mathrm{~W}$, with $15 \mathrm{~W}$ increases every $1 \mathrm{~min}$ ) until they reached $80-85 \%$ of the agepredicted maximal heart rate $(208-$ age $\times 0.7)$. A prediction of $V \mathrm{O}_{2 \max }$ was made by the extrapolation of the linear regression line between heart rate and oxygen consumption during cycle exercise and the age-predicted maximum heart rate [10,11].

Exercise training intervention. All subjects underwent a supervised orientation and thereafter performed open-air aerobic exercise training (brisk walking/jogging) for 16 weeks. During the first 2 weeks, subjects performed a relatively low-intensity exercise training $(60 \%$ of heart rate reserve, $30 \mathrm{~min} /$ day, $2-3$ days/week). Thereafter the exercise intensity as well as the duration of training were increased $(75 \%$ of heart rate reserve, $45 \mathrm{~min} /$ day, 3-4 days/week). Individual heart rate reserve was evaluated by the Karvonen formula. The resting heart rate at the measurement before the training and the age-predicted maximal heart rate were used for resting and maximal heart rate for the heart rate reserve calculation. The intensity of the exercise training was monitored by a Polar heart rate monitor and physical activity logs. The subjects were instructed to perform only scheduled exercise training during the training period. Restriction meals (calorie ingestion) were not carried out throughout the observation. All subjects were able to complete the training (59 sessions).

Statistical analysis. The data were analyzed using two-way (the training and arterial site) ANOVA with repeated measures. In the case of a significant $F$ value, a post hoc test using the Newman-Keuls method identified a significant difference from the value 
PWV

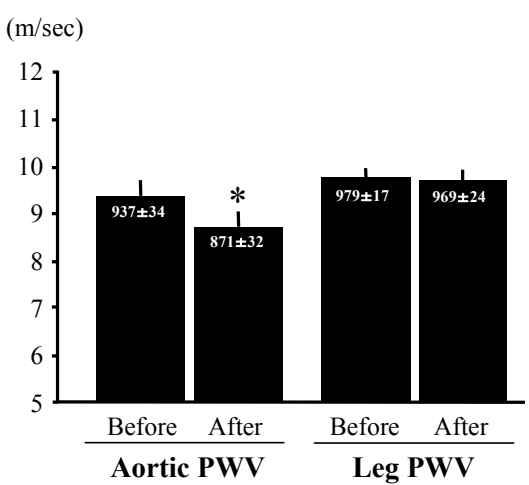

Arterial compliance

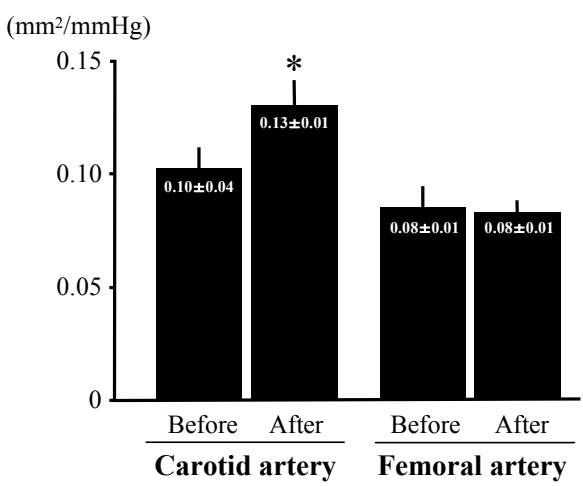

Diastolic lumen Diameter

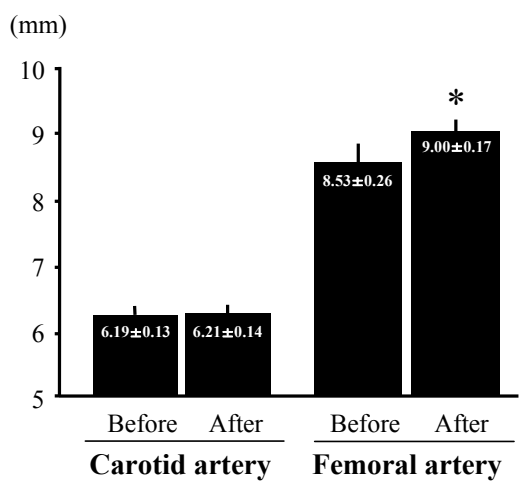

Fig. 1. Aortic and leg PWV (left), carotid and femoral arterial compliance (center), and diastolic lumen diameter (right) before and after the exercise-training intervention. ${ }^{\star} p<0.05$ vs. before training.

before training. All data are reported as mean \pm SE. Statistical significance was set at $P<0.05$ for all comparisons.

\section{Results}

Body mass and resting heart rate significantly decreased after the training program (both $P<0.05$, Table 1). Brachial and carotid arterial pressures did not change significantly (Table 1). Tibial systolic, diastolic, and mean arterial pressure significantly decreased after the training, but tibial pulse pressure did not change (Table 1). The estimated $V \mathrm{O}_{2 \max }$ increased significantly after the training program $(P<0.05)$. Following the training program, the femoral arterial diastolic lumen diameter significantly increased, but carotid arterial diastolic lumen diameter did not change (Fig. 1). An interaction effect of the training and the arterial site was not significant $(P=0.07)$. Aortic PWV significantly decreased $(P<0.05)$, but leg PWV did not change (Fig. 1). Carotid arterial compliance significantly increased, but femoral arterial compliance did not change significantly (Fig. 1). These interaction effects of the training and the arterial site were statistically significant (both $P<0.05$ ).

\section{Discussion}

Primary findings of the present study are as follows. Central arterial stiffness reduced after the exercise training intervention, but peripheral arterial stiffness did not change significantly. There was a significant interaction effect of the exercise training and the arterial site. These results suggest that the stiffness of the peripheral artery is difficult to change in shortterm and moderate-intensity aerobic exercise training, which is able to significantly improve central arterial stiffness in middle-aged healthy men.

High-intensity physical training induces the expansion in peripheral conduit artery $[5,12]$. According to the Moen-Korteweg equation [1], the increased arterial diameter is partly associated with the reduction in arterial stiffness. Indeed, Schmidt-Trucksuss et al. [5] indicated that femoral arterial diameter and compliance in endurance-trained males (e.g., cyclists, middle- or long-distance runners, and triathletes) were significantly larger than those in age-matched sedentary counterparts. A previous interventional study [12] showed that 6 weeks of high-intensity single-legged cycling training $(80 \%$ of one-legged peak oxygen consumption) induced a significant expansion in the femoral arterial cross-sectional area in the trained leg. Even moderate-intensity aerobic exercise training could increase femoral arterial diameter [6]. Similarly, in the present study femoral arterial diameter expanded significantly after the exercise training, whereas leg PWV and femoral arterial compliance did not change significantly. Therefore, although the expansion of the peripheral conduit artery appears to be induced by even a moderate-intensity exercise training program, it may not lead to a reduction in its stiffness.

It is unclear what physiological mechanisms explain the site specificity of the improvement of arterial stiffness with aerobic exercise training. We can, however, only speculate on the lying mechanism(s). The relative amount of elastin of the fibrous elements of the arterial wall is greater in central elastic arteries than in peripheral muscular arteries, and the age-related histological changes in the arterial wall (i.e., a decrease in elastin and the proliferation of collagen fiber in media), the main factor of arterial stiffening with age, are more rapid in central elastic arteries 
Table 1. Physiological characteristics before and after training.

\begin{tabular}{lcc}
\hline \multicolumn{1}{c}{ Variables } & Before training & After training \\
\hline Body mass $(\mathrm{kg})$ & $68 \pm 3$ & $67 \pm 3^{*}$ \\
Heart rate $(\mathrm{bpm})$ & $58 \pm 2$ & $53 \pm 1^{*}$ \\
Brachial systolic blood pressure $(\mathrm{mmHg})$ & $119 \pm 3$ & $115 \pm 3$ \\
Brachial diastolic blood pressure $(\mathrm{mmHg})$ & $76 \pm 3$ & $74 \pm 3$ \\
Brachial mean arterial pressure $(\mathrm{mmHg})$ & $90 \pm 3$ & $87 \pm 3$ \\
Brachial pulse pressure $(\mathrm{mmHg})$ & $43 \pm 1$ & $41 \pm 1$ \\
Carotid systolic blood pressure $(\mathrm{mmHg})$ & $109 \pm 3 \pm 3$ \\
Carotid pulse pressure $(\mathrm{mmHg})$ & $33 \pm 1$ & $32 \pm 1$ \\
Tibial systolic blood pressure $(\mathrm{mmHg})$ & $142 \pm 4$ & $136 \pm 4^{*}$ \\
Tibial diastolic blood pressure $(\mathrm{mmHg})$ & $77 \pm 2$ & $72 \pm 3^{*}$ \\
Tibial mean arterial pressure $(\mathrm{mmHg})$ & $96 \pm 3$ & $90 \pm 4^{*}$ \\
Tibial pulse pressure $(\mathrm{mmHg})$ & $65 \pm 2$ & $65 \pm 3$ \\
Estimated VO ${ }_{2 \text { max }}$ (ml/kg/min) & $32 \pm 1$ & $34 \pm 1^{*}$ \\
\hline
\end{tabular}

bpm $=$ beats per minute. ${ }^{*} p<0.05$ vs. before training. Data are expressed as mean $\pm \mathrm{SE}$.

than in peripheral arteries [1]. Similarly, in previously sedentary middle-aged people, it is plausible that the magnitude of the training-related improvement of central arterial stiffness may be greater than that of the peripheral artery. It was shown that 16 weeks of aerobic exercise training reduced the calcium content of elastin and increased the elastin content in the aortic wall in rats [13]. Such differential effects of aging on central and peripheral arterial stiffness may explain the present results that moderate-intensity exercise training reduces central arterial stiffness, but not peripheral arterial stiffness. Alternatively, the difference in vessel wall distensibility between the central and peripheral arteries may be related to the site specificity of the improvement of arterial stiffness with exercise training. Using cultured endothelial cells in an artificial tube and a servo-controlled perfusion system, Peng et al. [14] demonstrated that an augmentation of endothelial Akt/eNOS phosphorylation at a given pulse pressure and shear stress is greater in a compliant tube than in a stiffer tube, implying that wall compliance (magnitude of cyclic stretch) itself affects the training-induced change in arterial stiffness via mechano-biochemical signaling compromising vasorelaxation. Further studies will be necessary to determine the physiological precise underlying mechanisms.

In summary, we tested the hypothesis that in middle-aged men a short-term aerobic-exercise training (walking/jogging) reduces arterial stiffness in the lower extremities. After the training intervention, PWV significantly decreased in the aorta, but not in the leg.
Arterial compliance significantly increased in the carotid artery, but not in the femoral artery. These interaction effects of the training and the arterial site were significant. The results suggest that the stiffness of the peripheral artery may be difficult to change with aerobic exercise training, which is able to sufficiently decrease central arterial stiffness in middle-aged men.

We would like to thank Eiko Yamamoto and Keiko lemitsu for their assistance in the present study.

\section{REFERENCES}

1. Nichols W and O'Rourke M: McDonald's Blood Flow in Arteries: Theoretical, Experimental and Clinical Principles, Aenord, London, 1998

2. Blacher J, Guerin AP, Pannier B, Marchais SJ, Safar ME, and London GM: Impact of aortic stiffness on survival in end-stage renal disease. Circulation 99: 2434-2439, 1999

3. Tanaka $H$, Dinenno FA, Monahan KD, Clevenger CM, DeSouza CA, and Seals DR: Aging, habitual exercise, and dynamic arterial compliance. Circulation 102: 1270-1275, 2000

4. Tanaka H, DeSouza CA, and Seals DR. Absence of age-related increase in central arterial stiffness in physically active women. Arterioscler Thromb Vasc Biol 18: 127-132, 1998

5. Schmidt-Trucksass A, Schmid A, Brunner C, Scherer N, Zach G, Keul J, and Huonker M: Arterial properties of the carotid and femoral artery in endurance-trained and paraplegic subjects. J Appl Physiol 89: 1956-1963, 2000

6. Dinenno FA, Tanaka H, Monahan KD, Clevenger CM, Eskurza I, DeSouza CA, and Seals DR: Regular endur- 
ance exercise induces expansive arterial remodelling in the trained limbs of healthy men. J Physiol 534: 287 295, 2001

7. Kingwell BA, Berry KL, Cameron JD, Jennings GL, and Dart AM: Arterial compliance increases after moderate-intensity cycling. Am J Physiol 273: H2186-H2191, 1997

8. Sugawara J, Hayashi K, Yokoi T, Cortez-Cooper MY, Devan AE, Anton MA, and Tanaka H: Brachial-ankle pulse wave velocity: an index of central arterial stiffness? J Hum Hypertens 19: 401-406, 2005

9. Miyachi M, Kawano H, Sugawara J, Takahashi K, Hayashi K, Yamazaki K, Tabata I, and Tanaka H: Unfavorable effects of resistance training on central arterial compliance: a randomized intervention study. Circulation 110: 2858-2863, 2004

10. Fitchett MA: Predictability of VO2 max from submaxi- mal cycle ergometer and bench stepping tests. $\mathrm{Br} J$ Sports Med 19: 85-88, 1985

11. Maritz J, Morrison J, Peter J, Strydom N, and Wyndham C: A practical method of estimating an individual's maximal oxygen intake. Ergonomics 4: 97-122, 1961

12. Miyachi M, Tanaka H, Yamamoto K, Yoshioka A, Takahashi K, and Onodera S: Effects of one-legged endurance training on femoral arterial and venous size in healthy humans. J Appl Physiol 90: 2439-2444, 2000

13. Matsuda M, Nosaka T, Sato M and Ohshima N: Effects of physical exercise on the elasticity and elastic components of the rat aorta. Eur J Appl Physiol Occup Physiol 66: 122-126, 1993

14. Peng X, Haldar S, Deshpande S, Irani K, and Kass DA: Wall stiffness suppresses Akt/eNOS and cytoprotection in pulse-perfused endothelium. Hypertension 41: 378-381, 2003 